\title{
Transparent conducting, anti-static and anti-static-anti-glare coatings on plastic substrates
}

\author{
N. Al-Dahoudi, H. Bisht, C. Göbbert, T. Krajewski, M.A. Aegerter* \\ Institut für Neue Materialien - INM, Department of Coating Technology, Im Stadtwald, Gebäude 43, 66123 Saarbrücken, Germany
}

\begin{abstract}
In $\mathrm{O}_{3} \mathrm{Sn}$ (ITO) sols made of crystalline nanoparticles, fully redispersable in an ethanol solution containing hydrolyzed organosilanes, have been developed to deposit conducting transparent and anti-glare coatings on plastic (PMMA, polycarbonate) and glass substrates by spin, dip and spray coating processes. The coatings are cured by UV irradiation and/or by a low temperature heat treatment $\left(T=130^{\circ} \mathrm{C}\right)$ in air or reducing atmosphere. The electrical, optical, textural and mechanical properties of the coatings are reported. A stable sheet resistance as low as $5 \mathrm{k} \Omega_{\square}$, was obtained with a single 500 -nm thick transparent layer. Anti-glare anti-static coatings exhibiting a $40-\mathrm{k} \Omega \Omega_{\square}$ sheet resistance, a gloss of $60-70 \mathrm{GU}$, a clarity of $75-90 \%$ and an optical resolution $>8$ lines $/ \mathrm{mm}$ were obtained by a room temperature spraying process. The abrasion resistance of both coatings is in agreement with DIN 58196 -H25-class 1.
\end{abstract}

Keywords: Sol-gel; Coatings; Conductivity; Indium tin oxide; Plastic substrate

\section{Introduction}

Transparent conducting inorganic ( $\mathrm{TCO}$ ) coatings are today widely used as electrodes in optoelectronic devices (displays, smart windows and mirrors, etc.), as IR reflecting layers in low-emissivity glazings and oven windows, as heatable layers in defrosting windows, for electromagnetic shielding or dissipating static, etc. The most important materials bearing such properties are very thin metal layers ( $\mathrm{Ag}, \mathrm{Au}$, etc.) and $n$-type oxide semiconductors such as indium tin oxide (ITO), fluorine or antimony doped tin dioxide (FTO, ATO) or aluminium or gallium doped zinc oxide (AZO, GZO) [1]. The deposition of such layers using CVD, AACVD, spray pyrolysis and conventional sol-gel techniques requires either a hot substrate $\left(T>400^{\circ} \mathrm{C}\right)$, or a sub-

\footnotetext{
* Corresponding author. Tel.: +49-681-9300-317; fax: +49-6819300-249.

E-mail address: aegerter(a inm-gmbh.de (M.A. Aegerter).
}

strate which can be heated after the deposition to such a high temperature. These processes are, therefore, only adequate to coat glasses [2].

There is today a great interest in coating materials and devices which do not withstand high temperature, such as plastic substrates or preformed glasses. For that purpose, only physical deposition techniques are adequate [2]. Wet coating deposition (spray, dip and spin coating) are also, in principle, well-adapted techniques to coat small to large substrates with various geometries using a relatively cheap infrastructure, as new sol formulations incorporating organic precursors (organic-inorganic hybrids) allow us to obtain thick coatings fully processable at low temperatures $(T<$ $\left.150^{\circ} \mathrm{C}\right)$.

For the development of TCO coatings, one possible issue is the use of hybrid sols containing a high amount of already conducting crystalline oxide nanoparticles. The preparation of anti-static transparent and anti-glare ITO based coatings with a sheet resistance $R_{\square} \geq 100$ $\mathrm{k} \Omega_{\square}$ has been recently reported by us [3].

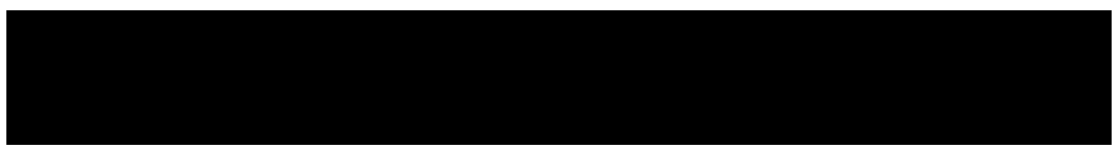


This paper reports on recent progress obtained in the preparation of such coatings and their optical, electrical, mechanical and textural characterization.

\section{Experimental}

\subsection{Precursors and sols}

\subsubsection{ITO particle preparation and characterization}

$\mathrm{In}_{2} \mathrm{O}_{3}: \mathrm{Sn}$ powders were prepared by a controlled growth technique [4,5]. A solution of $0.5 \mathrm{~mol}$ indium(III) chloride in $1000 \mathrm{ml}$ ethanol containing $8 \mathrm{~mol} \%$ of $\mathrm{SnCl}_{4}$ (with respect to In) was added dropwise to 100 $\mathrm{ml}$ of an aqueous ammonia solution $(25 \mathrm{wt} . \%$ ) containing $14 \mathrm{~g}(10 \mathrm{wt} . \%$ with respect to the oxide) of $\beta$-alanine, a surface modifying agent. The ITO suspensions were treated at $80^{\circ} \mathrm{C}$ for $24 \mathrm{~h}$ under atmospheric pressure. The resulting powders were isolated by centrifugation, washed with water several times until the measured ionic conductivity reached a value smaller than 20 $\mu \mathrm{S} / \mathrm{cm}$. The powder was first dried at $60^{\circ} \mathrm{C}$ and then annealed at $250^{\circ} \mathrm{C}$ in a reducing atmosphere containing $\mathrm{N}_{2}$ and $\mathrm{H}_{2}$ in a volume ratio of 90 to $10 \%$.

The powders were characterized by high resolution transmission electron microscopy (HRTEM CM200 FEG, Philips) and X-ray diffraction (D500, Siemens). Their crystallite size was calculated using the Scherrer equation.

\subsubsection{Nanoparticles solution}

The dried ITO powder was first mechanically redispersed in ethylene glycol with a carbon acid as dispersion agent. The solution was then added to ethanol or water. Both suspensions showed a blue color and are stable (no precipitation) with solid contents up to 40 wt.\% over several months. For the zeta potential and the hydrodynamic particle size (Ultrafine Particle Analyzer, UPA), $0.1 \mathrm{~g}$ of the powders were dispersed in 10 $\mathrm{ml} \mathrm{H}_{2} \mathrm{O}$ using ultrasonic radiation. The $\mathrm{pH}$ was adjusted to 2 with $\mathrm{HNO}_{3}$. The zeta potential curves (Zetasizer, Malvern) were measured in a $\mathrm{pH}$ range from 2 to 12 using a microelectrophoresis cell.

\subsubsection{Transparent conducting sol}

An ethanolic solution containing 25 wt.\% redispersed ITO nanoparticles (see above) was modified by adding various amount of hydrolyzed 3-glycidoxypropyltrimethoxysilane (GPTS) or 3-methacryloxypropyltrimethoxysilane (MPTS) under an ultrasonic bath. PMMA, polycarbonate (PC) and glass substrates were coated by spin and dip coating process. The resulting films were cured in air or reducing atmosphere at $130^{\circ} \mathrm{C}$ up to $20 \mathrm{~h}$, by UV irradiation with an average intensity of $105 \mathrm{~mW} / \mathrm{cm}^{2}$ up to $110 \mathrm{~s}$ (Beltron) or by a combination of both treatments.

\subsubsection{Anti-glare conducting sols}

An ITO nanoparticles solution ( $25 \mathrm{wt} . \%$ ) was modified by adding GPTS. The sols were sprayed at room temperature for a period of $15-20 \mathrm{~s}$ using a SATA mini-jet spray gun $(0.5 \mathrm{~mm}$ nozzle, $3 \mathrm{bar})$ on polycarbonate or glass substrates. The coatings, a few micrometers thick, were polymerized by UV irradiation (105 $\mathrm{mW} / \mathrm{cm}^{2}, 110 \mathrm{~s}$ ).

\subsection{Characterization of the coatings}

\subsubsection{Textural properties}

The average roughness of the coatings was determined using a Tencor P10 stylus profilometer. The morphology of coating cross sections was observed by SEM (JEOL 6400) and HR-TEM (Philips, $200 \mathrm{keV}$ ).

\subsubsection{Optical properties}

Haze, clarity and gloss were measured using a ByK Gardner plus and a micro-TRI reflectometer. Optical transmission and reflection were determined using a Variant Cary $5 \mathrm{E}$ spectrophotometer in the wavelength range $300-3000 \mathrm{~nm}$.

\subsubsection{Mechanical properties}

The abrasion resistance of the coatings was tested according to DIN 58196- $\mathrm{H} 25$ - rubbing with a cloth under a load of $9.8 \mathrm{~N}$ (instead of $4.9 \mathrm{~N}$ ), and DIN 58196-G10 - rubbing with an eraser under a load of $9.8 \mathrm{~N}$. The thickness $(t)$ was determined using a Tencor P10 profilometer.

\subsubsection{Electrical properties}

The sheet resistance $\left(R_{\square}\right)$ was measured by a fourpoint technique and the resistivity calculated from $\rho=$ $R_{\square} \cdot t$.

\section{Results and discussion}

\subsection{ITO nanoparticles and suspensions}

The ITO particles exhibit a cubic $\operatorname{In}_{2} \mathrm{O}_{3}$ phase. Phase separation and the orientation of the crystallites were

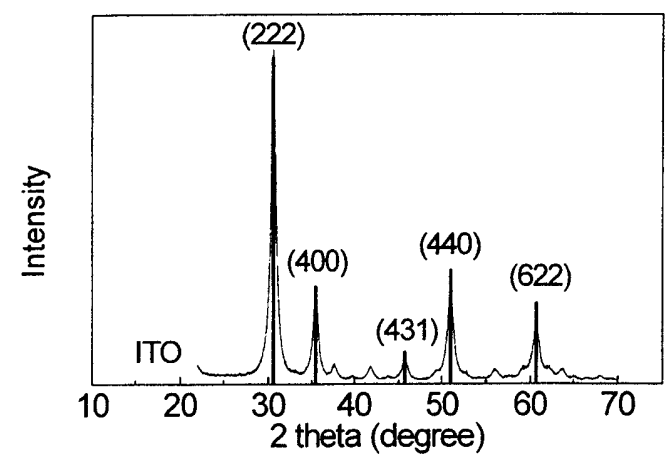

Fig. 1. XRD of dried and annealed nanoscaled $\mathrm{In}_{2} \mathrm{O}_{3}: \mathrm{Sn}$ powder. The vertical lines correspond to the JCPDS 06-416 database (cubic indium oxide structure). 


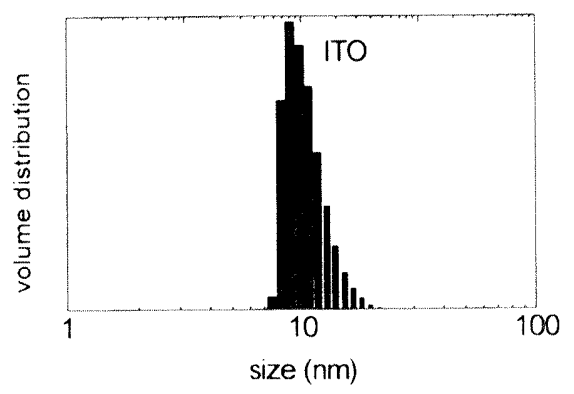

Fig. 2. ITO hydrodynamic particle size distribution in the suspension

not detected (Fig. 1). The crystallite size determined from the (222) peak is $15 \mathrm{~nm}$. Their specific surface area is $67 \mathrm{~m}^{2} / \mathrm{g}$ and their density is $6.45 \mathrm{~g} / \mathrm{cm}^{3}$, corresponding to $92 \%$ of the theoretical density of $\mathrm{In}_{2} \mathrm{O}_{3}[5]$.

The stability of the ethanolic dispersion is strongly dependent on the pH of the solvent. From the $\zeta$ potential determination, the isoelectric point of the surface modified ITO particles was found to be $\mathrm{pH}_{\text {icp }}$ $=8.5$ and the suspension was stable at $\mathrm{pH}>6$. A typical hydrodynamic size distribution of the particles is shown in Fig. 2.

The average value is in agreement with that determined from the X-ray measurement. The particles are, therefore, fully dispersed down to the primary particle size and each particle is formed by a single crystallite. This has been also confirmed by HRTEM (Fig. 3) where no evidence of agglomeration is observed and an average particle size of $15-20 \mathrm{~nm}$ can be determined.

\subsection{Transparent conducting coating}

The evolution of the sheet resistance of a $500-\mathrm{nm}$ thick single layer MPTS/ITO coatings deposited on a 3-mm thick PC substrate is shown in Fig. 4 as a function of the sol composition for different annealing treatments. Similar overall behavior has been obtained with GPTS/ITO coatings. Whatever the treatment is,

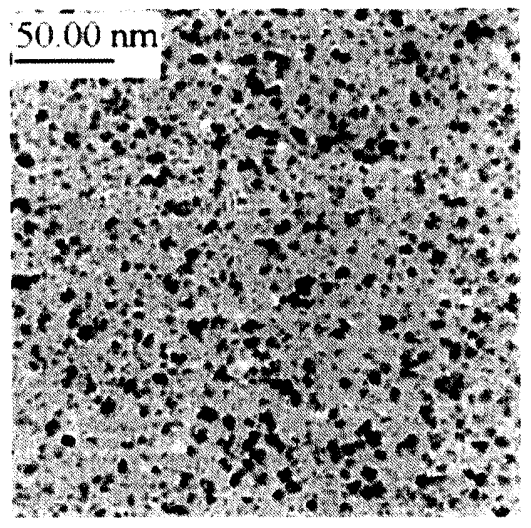

Fig. 3. HRTEM picture of crystalline $\mathrm{In}_{2} \mathrm{O}_{3}: \mathrm{Sn}$ nanopowder after annealing and redispersion in ethanol $(\mathrm{pH}=4)$.

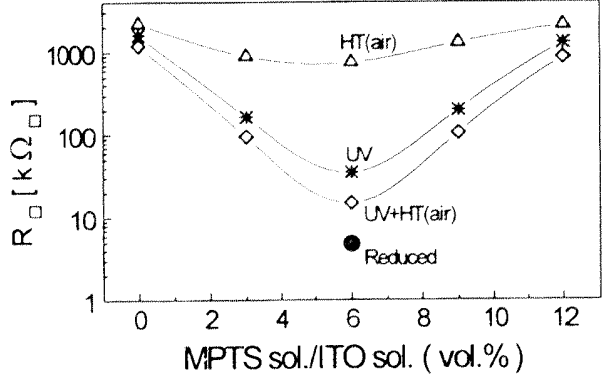

Fig. 4. Sheet resistance of $500-\mathrm{nm}$ thick MPTS/ITO coatings vs. sol composition. UV: UV irradiation $105 \mathrm{~mW} / \mathrm{cm}^{2}, 110 \mathrm{~s}$. HT (air) Heat treatment in air: $130^{\circ} \mathrm{C}, 15 \mathrm{~h}$.

$R_{\mathrm{O}}$ for pure ITO is high ( $>1 \mathrm{M} \Omega_{\square}$ ) and the coating adhesion is poor (DIN 58196-H25 class 5). Cured MPTS/ITO coatings present a minimum of the sheet resistance for a composition volume ratio of $6 \%$, and at and above this ratio, they do not exhibit abrasion damage according to DIN 58196-H25 (class 1) and only slight scattering according to DIN 58196-G10 (class 2). The lowest stable sheet resistances, $R_{\square}=5 \mathrm{k} \Omega_{\square}$, for MPTS /ITO and $R_{\square}=15 \mathrm{k} \Omega_{\square}$ for GPTS /ITO, have been obtained for a 110-s UV irradiation followed by a $15-\mathrm{h}$ heat treatment at $130^{\circ} \mathrm{C}$ with further annealing in a reducing atmosphere.

The values reported above have been measured in air at least one week after the coating's production. They are, however, strongly dependent on the type of atmosphere. Fig. 5 shows the time evolution $R_{\square}(t)$ measured for 500-nm thick GPTS /ITO coatings left in vacuum, in air (approx. 50\% RH) and in water. Similar overall behavior was obtained with MPTS/ITO coatings. Immediately after the coating production, the $R_{\square}$ values are smaller than those reported above: $7 \mathrm{k} \Omega$ for GPTS-ITO and $1.3 \mathrm{k} \Omega$ for MPTS/ITO. They remain constant in vacuum, but steadily increase in air and water. They reached a maximum stable value after approximately 7 days.

The mechanisms leading to these variations are not yet clear, but seem to be related to the composition and the morphology of the coatings. FTIR transmission

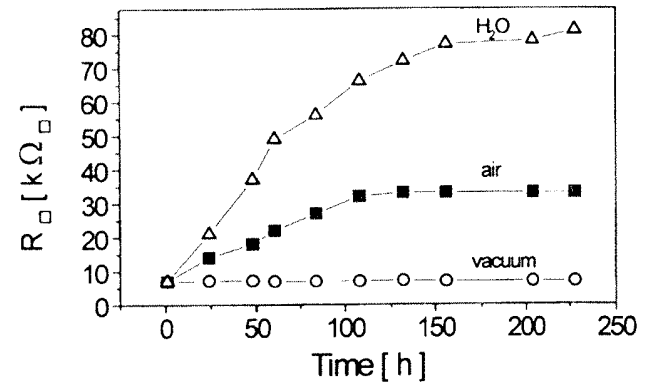

Fig. 5. Time evolution of the sheet resistance of 500-nm thick GPTS /ITO $(6$ vol. $\%)$ coatings left in vacuum, air and water. The coatings have been annealed in UV light $\left(105 \mathrm{~mW} / \mathrm{cm}^{2}, 110 \mathrm{~s}\right)$ followed by a 15 -h heat treatment in air at $130^{\circ} \mathrm{C}$. 
spectra of MPTS/TO coatings deposited on the $\mathrm{Si}$ wafer and annealed by UV irradiation or heat treatment are different (Fig. 6). The broad absorption observed between 4000 and $1000 \mathrm{~cm}^{-1}$ is common to both spectra and is related to the ITO content. Contrary to what is observed for pure MPTS coatings, the heat treatment does not completely eliminate the $\mathrm{C}=\mathrm{C}$ band $\left(1636 \mathrm{~cm}^{-1}\right)$ and only slightly reduced the $\mathrm{C}=\mathrm{O}$ band at $1716 \mathrm{~cm}^{-1}$, and a well defined $\mathrm{Si}-\mathrm{O}-\mathrm{Si}$ network $\left(1050 \mathrm{~cm}^{-1}\right)$ is not observed. The presence of ITO particles seems to impede the polymerization and condensation processes. On the contrary, the UV treatment eliminates the $\mathrm{C}=\mathrm{C}$ band, strongly reduces the $\mathrm{C}=\mathrm{O}$ band, and a well defined $\mathrm{Si}-\mathrm{O}-\mathrm{Si}$ network is found to lead to a more homogeneous particle binding material.

The morphology of the coatings are also different (Fig. 7). The coating cured by heating is built of loosely packed globular particles, $40-80 \mathrm{~nm}$ in size. The UV cured layer has a smaller thickness and appears more homogeneous and denser. This is confirmed by the TEM cross section (Fig. 8) which shows a dense pack-

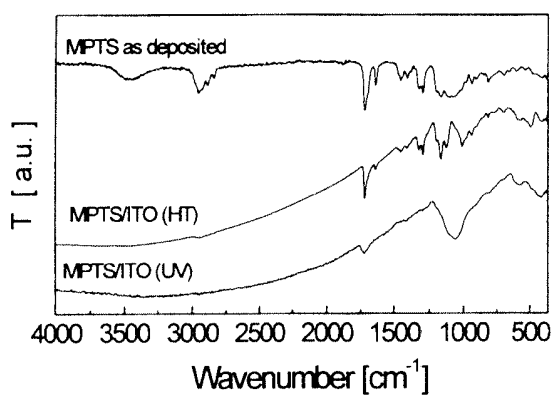

Fig. 6. IR spectra of pure MPTS (as deposited) and MPTS/ITO coatings deposited on a Si wafer. HR: Heat treated in air at $130^{\circ} \mathrm{C}$, 15 h., UV: UV irradiated at $105 \mathrm{~mW} / \mathrm{cm}^{2}, 110 \mathrm{~s}$.

ing of $10-15-\mathrm{nm}$ sized ITO particles (left picture), already crystalline (right picture). We believe that the lower sheet resistance obtained by UV treatment is due to a slightly smaller inter-particle separation. A further treatment in reducing atmosphere still decreases the resistivity (as is usual for ITO material). The value is still relatively high compared to sputtered coatings. This certainly comes from the high electronic scatter-
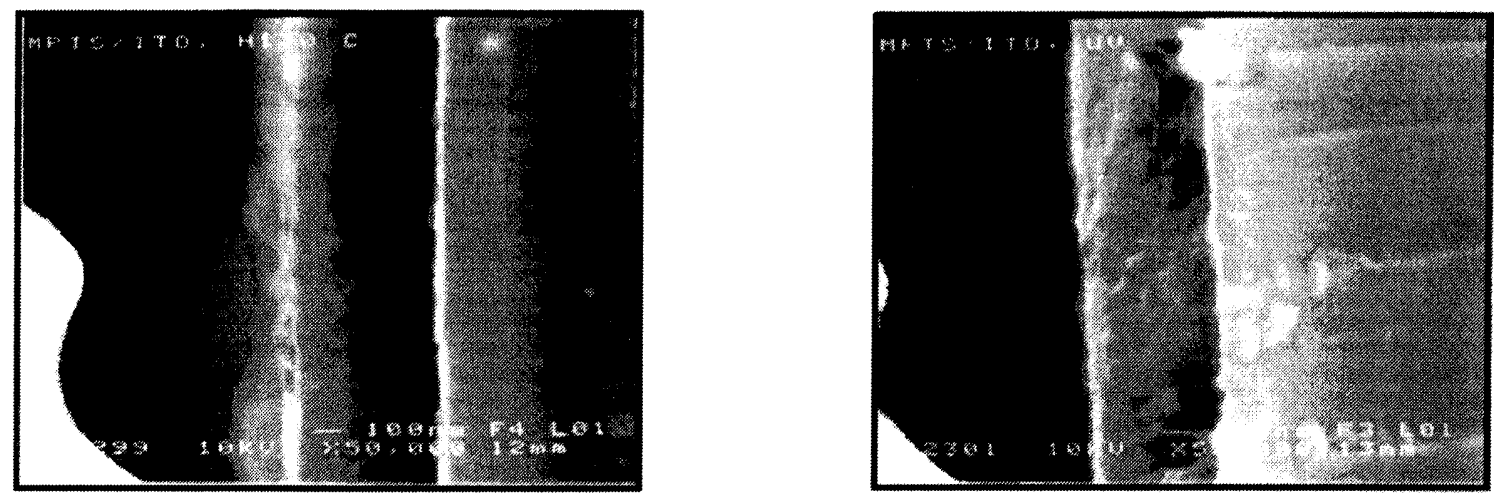

Fig. 7. SEM pictures of a cross-section of MPTS/ITO coatings heat treated at $130^{\circ} \mathrm{C}, 15 \mathrm{~h}$ (left) and $110 \mathrm{~s} \mathrm{UV} \mathrm{cured} \mathrm{(right).}$
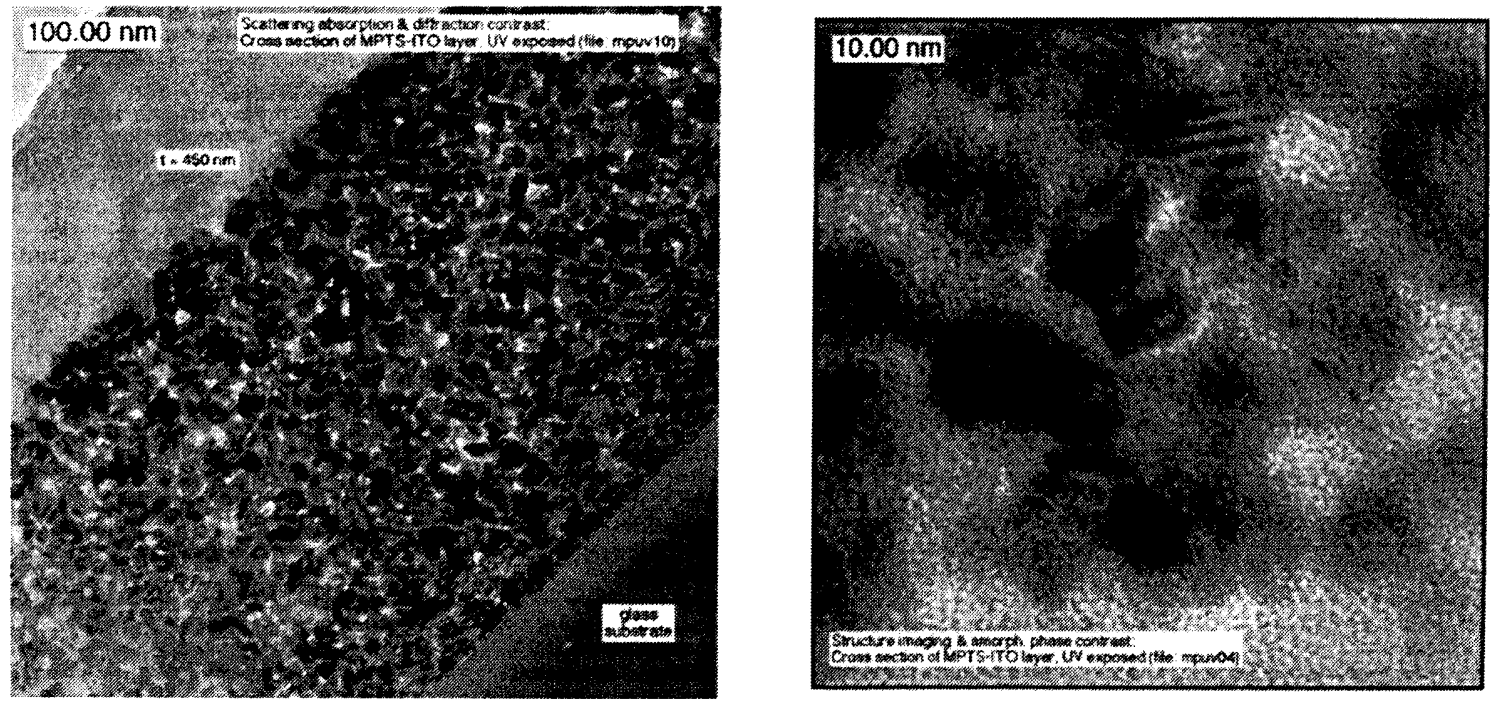

Fig. 8. TEM pictures of a cross-section of a UV cured MPTS/ITO coating with different magnification. 


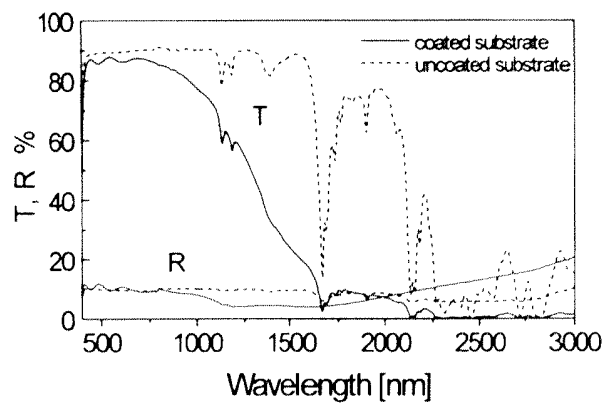

Fig. 9. UV-near IR transmission $(T)$ and reflection $(R)$ measured against air of a $500 \mathrm{-nm}$ thick GPTS/ITO coating deposited on a $3-\mathrm{mm}$ thick polycarbonate (PC) substrate and an uncoated PC substratc.

ing due to the very small size of the particles. Tests are underway with larger redispersable ITO particles.

The optical transmission and reflection spectra of a 3-mm thick uncoated PC substrate and one coated with a 500-nm GPTS /ITO layer are shown in Fig. 9. A high transmission of approximately $87 \%$ is observed in the visible range. The influence of the carrier is clearly seen by the strong absorption occurring in the near IR range $(900 \mathrm{~nm}<\lambda<2000 \mathrm{~nm})$ and the increase of the reflection for $\lambda>2 \mu \mathrm{m}$.

\subsection{Anti-glare conducting coatings}

The spray gun delivers droplets with average size of $25 \mu \mathrm{m}$. When arriving on the cold substrate, they spread and form a rough surface which can be polymerized by UV irradiation or by heating at $T \approx 130^{\circ} \mathrm{C}$. The thickness of the coatings is in the range of a few $\mu \mathrm{m}$.

XRD measurements are in agreement with the random cubic structure shown in Fig. 1 with crystallite size reflecting the size of the ITO particles $(15 \mathrm{~nm})$. Fig. 10 shows the surface morphology of such coatings observed with an optical microscope. The size of the surface features varies from approximately $10-100 \mu \mathrm{m}$ and the average roughness of the coatings measured by profilometer along a $500-\mu \mathrm{m}$ length is $R_{a}=0.2 \mu \mathrm{m}$ with height variation of $\pm 0.4 \mu \mathrm{m}$. The sheet resistance is approximately $150 \mathrm{k} \Omega \Omega_{\square}$. This value can be lowered to approximately $30 \mathrm{ks} \Omega_{\square}$ if a transparent anti-static ITO underlayer is first deposited (see Section 3.2). Table 1 shows the results of the optical and abrasion properties of the coatings.

Fig. 11 shows the optical effect of such coatings. The image (entrance of INM) placed $2 \mathrm{~cm}$ behind a coated and a non-coated substrate is clearly visible and the

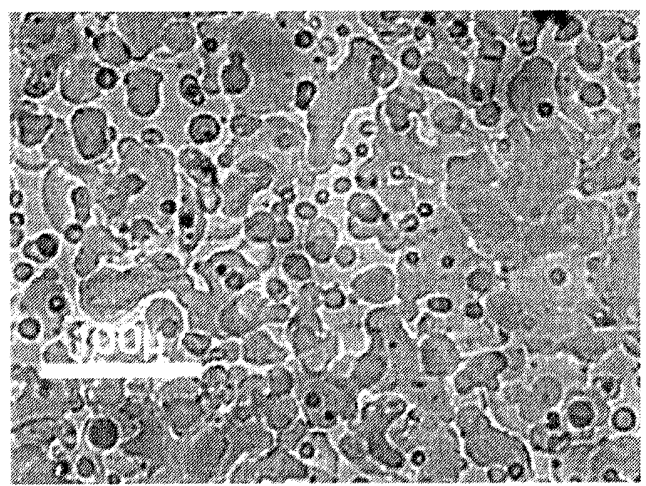

Fig. 10. Surface morphology of antiglare coatings sprayed at room temperature on a PC substrate and UV polymerized.

glare of the white object placed in front of the substrates is strongly reduced at the coated side.

\section{Conclusions}

Stable hybrid sols allowing the deposition of conducting, anti-static and anti-glare-anti-static coatings fully processable at low temperature have been developed. They are obtained by modifying an ethanolic suspension of dispersed crystalline ITO nanoparticles with hydrolyzed silanes (GPTS, MPTS). A 500-nm thick transparent single layer with a stable sheet resistance as low as $5 \mathrm{k} \Omega_{\mathrm{a}}$, and abrasion resistance in agreement with DIN 58196-H25 class 1 and G 10 class 2 have been obtained by spin or dip coating processes on plastics (PMMA, PC). The best curing process involved a UV

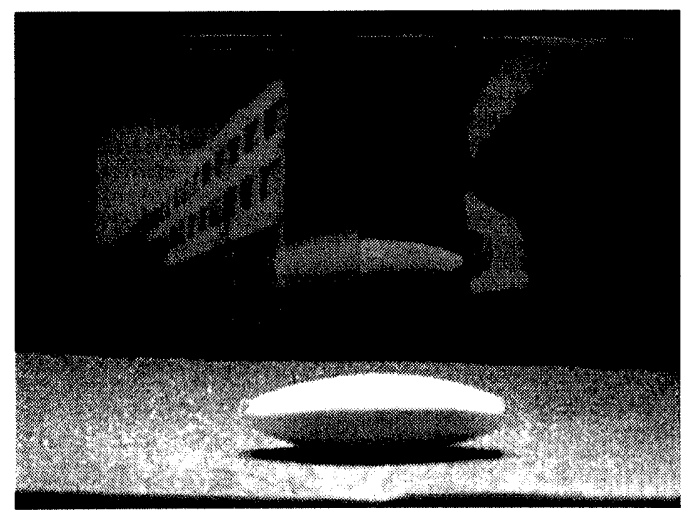

Fig. 11. Glaze of a white object placed in front of a AS-AG coated plastic (left) and an uncoated one (right). The picture of the building placed $2 \mathrm{~cm}$ behind the substrates is clearly visible in both configurations.

Table 1

Typical gloss, haze, clarity, resolution and abrasion of antiglare coatings deposited on plastic and glass substrates cured by UV irradiation

\begin{tabular}{lllll}
\hline Gloss $\left(a 0^{\circ}\right.$ & Haze $(\%)$ & Clarity $(\%)$ & $\begin{array}{l}\text { Resolution } \\
\text { (USAF chart) }\end{array}$ & Abrasion (9.8 N) \\
\hline $60-70$ & $\leq 10$ & $75-90$ & $\geq 8$ lines $/ \mathrm{mm}$ & DIN 58196-H25 \\
\hline
\end{tabular}


irradiation $\left(105 \mathrm{~mW} / \mathrm{cm}^{2}, 110 \mathrm{~s}\right)$ followed by a heat treatment at $T=130^{\circ} \mathrm{C}$ for $15 \mathrm{~h}$ and then a reducing treatment. Anti-static coatings with sheet resistance as low as $150 \mathrm{k} \Omega$, presenting an anti-glare effect $(\mathrm{GU} \approx$ 65) have been obtained on the same substrates by a spray process at room temperature followed by UV annealing.

\section{References}

[1] H.L. Hartnagel, A.L. Dawar, A.K. Jain, G. Jagdish, Semicon- ducting, Transparent Thin Film, IOP Publishing, Bristol and Philadelphia, 1995.

[2] H.K. Pulker, Coatings on Glass, 2nd ed, Elsevier, 1999.

[3] C. Goebbert, H. Bisht, N. Al-Dahoudi, R. Nonninger, M.A. Aegerter, H. Schmidt, J. Sol-Gel Sci. Technol. 19 (2000) 201-204.

[4] D. Burgard, C. Kropf, R. Nass, H. Schmidt, Better Ceramics Through Chemistry (1994) 101-107.

[5] C. Goebbert, R. Nonninger, M.A. Aegerter, H. Schmidt, Thin Solid Film 351 (1999) 79-84. 